Supporting Information

\title{
A New Machine-Learning Tool for Fast Estimation of Liquid Viscosity.
}

\section{Application to Cosmetic Oils}

Valentin Goussard $^{a}$, François Duprat $^{b^{*}}$, Jean-Luc Ploix ${ }^{b}$, Gérard Dreyfus $^{b}$, Véronique NardelloRataj $^{a}$ and Jean-Marie Aubry ${ }^{a^{*}}$

aUniversité de Lille, CNRS, ENSCL, UMR 8181, UCCS - Unité de Catalyse et de Chimie du Solide, 59655 Villeneuve d'Ascq, France

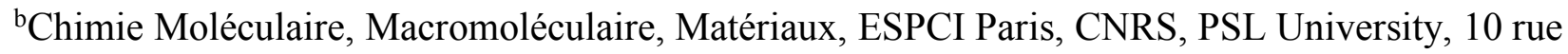
Vauquelin, 75005 Paris, France

*To whom correspondence should be addressed

Emails: jean-marie.aubry@univ-lille1.fr; arthur.duprat@espci.psl.eu 


\section{HOW TO LAUNCH THE DEMO}

In the following example the installation of Docker and the demo computations were made on a $3.2 \mathrm{GHz} 8$ Core Intel Xeon W iMac Pro with 64 GB of RAM running macOs Catalina 10.15.2.

1) Download the Docker application by copying and pasting the following line into a browser URL bar:

https://download.docker.com/mac/stable/Docker.dmg

2) After docker installation, launch it, open the docker preferences to increase the Docker allocated memory to $40 \mathrm{Go}$, and select all the 16 cores for the virtual machine (more or less according to the machine resources).

3) Open a terminal window, paste the following line, and hit return:

docker run -it --rm -v /docker:/host espcigm/viscosity:demo demo1

4) Once the 'espcigm/viscosity:demo' image has been completely downloaded, you can compare the SHA with the following genuine SHA:

sha25 6: 959894 dcba 739 f0bc 982 f c fe5d12bf2219a 99339968 dba $7803 b 030$ a 1 bad0ca $8 c$

5) The demo starts immediately when download is completed. You need to enter the path for the location of the docker directory in your home folder (typically/Users/home/docker, where home is the login name shortcut).

6) When the computation is done, you can open the excel file located in the folder/Users/home/docker/result.

In case the computation takes too much time on the computer, the argument $-\mathrm{H} 3$ can be added at the end of the run command (to reduce the complexity of the model):

docker run -it --rm -v /docker:/host espcigm/viscosity:demo demo1 -H 3

In addition, the viscosity value can be estimated for a single molecule ( $p$-xylene in the following example), by pasting the following command in a terminal window:

docker run -it --rm espcigm/viscosity: demo get ' $\mathrm{CC} 1=\mathrm{CC}=\mathrm{C}(\mathrm{C}) \mathrm{C}=\mathrm{C} 1^{\prime}$

More information can be obtained on how to download and install docker as well as how to use the demo in the next sections. 


\section{DOCKER CLIENT INSTALLATION}

\section{General}

Docker is a lightweight virtualization tool used to launch applications inside containers on Windows, MacOS or Linux computers; interactions with the host machine are fully under control, so that, after use, the host machine can be returned exactly to its original state. The use of Docker containers requires the installation of a local client, which depends on the host machine.

More information on how to install the local client on all machines can be found on the following pages:

https://docs.docker.com/engine/getstarted/step_one/

https://docs.docker.com/engine/installation/

\section{MacOS computer}

\section{Docker Client Installation}

The Macintosh computer should run at least Yosemite OS (Mac OS X.10.3) with a minimum of 4 GB of RAM (works only for computer manufactured after 2010).

Download the Docker application by copying and pasting the following line into a browser URL bar:

https://download.docker.com/mac/stable/Docker.dmg

Open the downloaded dmg file and drag the icon Docker.app onto the Applications icon as suggested. Launch the Docker application from the Applications folder to complete the installation (the system password is required). The presence of a whale icon in the top right status bar indicates that Docker is running and ready to receive commands via a terminal window.

For more information, visit:

https://docs.docker.com/engine/installation/mac/\#docker-for-mac

Note: If necessary, allocate more memory to the virtual machine using the Docker whale icon from the top right menu bar, then choosing preferences, moving the cursor to $3 \mathrm{~GB}$ (or more) and selecting restart. Make sure that the "Include VM in Time Machine backups" option is unselected in the General section of Docker preferences.

For more information visit the page:

https://docs.docker.com/engine/installation/mac/\#docker-for-mac

\section{Uninstalling Docker}

Access the Docker menu by clicking on the whale icon located on the finder top right menu bar, select the reset option, and then click on the uninstall box. Confirm this action with the Uninstall Docker choice within the next window. Once all the elements have been removed from the computer, drag the Docker application to the Trash and empty the latter.

\section{Linux computer}

\section{Docker Client Installation}

Installation instructions can be found for many Linux distributions on those pages:

- CentOS: https://docs.docker.com/engine/installation/linux/centos/

- Debian: https://docs.docker.com/engine/installation/linux/debian/

- Fedora: https://docs.docker.com/engine/installation/linux/fedora/

- Oracle Linux: https://docs.docker.com/engine/installation/linux/oracle/

- Red Hat: https://docs.docker.com/engine/installation/linux/rhel/

- Susa: https://docs.docker.com/engine/installation/linux/suse/

- Ubuntu: https://docs.docker.com/engine/installation/linux/ubuntu/ 
- Other Linux Distributions: https://docs.docker.com/engine/installation/linux/other/

Warning: depending on the chosen distribution and settings of the host computer, the requested commands might have to be entered in superuser mode, which is done with the "sudo" prefix at the beginning of the Docker command.

\section{Docker client installation on Ubuntu}

The Ubuntu Community Edition distribution is taken as an example.

\section{Prerequisites}

The ubuntu distribution must be one of the four 64-bit distributions:

- Zesty 17.4

- Xenial 16.04 (LTS)

- Trusty 14.04 (LTS)

- Bionic 18.04 (LTS)

Docker installation

- Update the apt package with the following command:

\$ sudo apt-get update

- Update Install the latest version of Docker with the command:

\$ sudo apt-get install docker-ce

- Check that Docker is installed correctly by downloading and running the image "hello-world":

\$ sudo docker run hello-world

\section{Uninstalling Docker and images}

Follow the instructions found at the uninstall Docker section of each distribution web page.

\section{Computer with Windows 10 or later}

\section{Docker Client Installation}

The computer must run at least Windows 10 64-bit Pro, Enterprise or Education (November 1511 Build 10586 or later) and have a minimum of 4 GB of RAM. The Hyper-V package must be activated. The Docker for Windows installer will activate it if necessary, which requires a reboot.

Download the Docker application with the following link:

https://download.docker.com/win/stable/InstallDocker.msi

Open the downloaded msi file for installation of the Docker application. Once installation is complete, Docker launches automatically. For more information visit the following page:

https://docs.docker.com/docker-for-windows/

Note: if necessary, more memory (and more CPUs) can be allocated for the computations; access the Docker whale icon in the task bar, choose preferences, and then increase the amount of memory (and the number of CPUs) used for Docker.

\section{Computer with Windows 7 and 8}

The Windows OS must be 64-bit, and virtualization must be enabled on the machine (activation to be done in the computer BIOS).

For more information about the virtualization support for Windows, visit:

http://docs.docker.com/toolbox/toolbox_install_windows/ 


\section{Installing the Docker Toolbox}

The latest Docker toolbox application (about $200 \mathrm{MB}$ ) can be downloaded from the page:

https://github.com/docker/toolbox/releases/

Start installing the Docker toolbox with the default values; the option "View Shortcuts in File Explorer" must be unselected. The Docker toolbox and virtualbox applications are installed in the "Program Files" directory of the C: drive, whereas the shortcuts for the "Docker Quickstart", "Oracle VM VirtualBox" and "Kinematic (Alpha)" applications are installed on the desktop.

Notes: 1) the Docker toolbox installation must be done within a session with administrator privileges; the choice is then given to install the application for the administrator or for all the computer users.

2) to avoid running out of memory during the docker compilation steps, a minimum of 4 GB memory is required for the docker-machine. This tuning can be done via the VM VirtualBox program. Choosing a maximum number of CPUs (or cores) will also speed up the computations to a large extent.

\section{Launching a test image in a Docker container}

Open a "Docker Toolbox" terminal by double-clicking the shortcut "Docker Quickstart". Wait for the prompt, which is the \$ sign. At the first launch, this process can take several minutes. To test that the Docker toolbox application is working properly, enter the following command:

docker run hello-world

If everything goes right the message "Hello from Docker!" appears in the terminal window, followed by some information about the client and the Docker daemon.

Note: using the Docker toolbox application requires a working internet connection.

\section{Uninstalling Docker and images}

The protocol detailed below uninstalls Docker and all its components.

Open a Docker toolbox terminal window and issue the command:

\section{\$ ls docker machine}

A list of files (xxxNamexxx) is returned by the list command.

Enter the following command to delete the matching image(s):

\$ rm xxxNamexxx docker machine

Note: the kinematic application can also be used to delete images.

Once the file(s) has(ve) been deleted, use the Windows uninstaller application (located in the control panel window) to remove the Docker toolbox application from the system.

For more information, visit the page:

https://www.docker.com/toolbox 


\section{GRAPH MACHINE AND MLP DEMONSTRATIONS WITH DOCKER CONTAINERS}

\section{Loading and launching the Docker image}

Open a terminal window (or a PowerShell window), type the following command and confirm it with the enter key (or copy and paste the text below):

docker pull espcigm/viscosity:demo

The image used to create containers is then downloaded. You can check that the image is genuine by checking that the hash code generated at the end of the download process is:

sha25 6: 959894 dcba 739 f0bc 982 f c fe 5 d12bf2219a 99339968 dba 7803 b0 030 albad0 ca 8 c

The same image is used to launch either graph machine or MLP computations. This image will be made available within 2 years of the manuscript publication date (April 6, 2020).

To open a container that will launch the default graph machine computations type the following line of text below (or copy and paste it):

docker run -it --rm -v /docker:/host espcigm/viscosity:demo demo1

To open a container that will launch the default MLP computations type the following line of text below (or copy and paste it):

docker run -it --rm -v /docker:/host espcigm/viscosity:demo demo2

At the end of the computations issued by one of these two commands, the results are written in a shared folder mounted inside the container. In the above command line, docker is the folder used; it is automatically created in the home folder at docker installation step $(\sim /$ docker $=/$ Users/home/docker on macOS, see below $)$. If this folder does not exist, no results are saved.

When typing one of the above two commands, you are asked to perform the following task:

"Please give the absolute path of the shared folder in the host"

Some suitable propositions for the absolute path that must be typed are given below for the Macintosh (Unix) and Windows OS, where 'home' is the home folder (or user folder), and 'docker' is the folder manually created in the home folder:

\section{/Users/' home'/docker \\ C: \Users\'home`\docker}

If, for any reason, the folder path has been incorrectly entered, you can correct this path name by deleting the file "host.pth" that is located inside the docker folder, and give the proper path at the next launch.

The demo computations are made with the graph machine and MLP numbers of hidden neurons chosen when looking for the appropriate complexity (i.e. eight and thirteen hidden neurons respectively). After completion of the demo, the container is automatically deleted. A new demo session can be started with the same command, but within a new container.

The excel file (with a xlsm extension) that is shared is recorded in the result subdirectory of the docker directory on the host machine ( / docker/result, where $\sim$ is a shortcut indicating the path to the connected user's home directory). The output filename is automatically incremented when the same command is executed.

Note: the above run commands are the minimal commands; if for example, the " $-\mathrm{v} \sim /$ docker: / host" is omitted in the command syntax, no excel file is created on the host machine, the computed results being lost when the container is deleted. The times (e.g. running time) reported during the demo depend on the machine used. On the contrary the VLOO score values for example should be the same when the run command is issued with the same parameters. See the Advanced Options section for more information about this run command. 
We explain hereafter a demo that describes the VLOO and the LOO computations; the command line for launching this demo is the following:

docker run -it --rm -v /docker:/host espcigm/viscosity:demo demo1 -H 3 -a train, test, $100-$ s 1908839970

\section{Explanations:}

This demonstration has four parts:

- Data analysis

- Creation of the training model and training

- Test on a fresh data set, different from the training data set.

- LOO cross-validation

\section{Data file analysis}

The data file used is the excel file BJMA300EMO.xlsx, that can be downloaded separately. The training data for the 300 molecules of the training set are contained in the DATA cell range. Each line of this file contains a molecule name, its SMILES code, the five computed $\sigma$-moments, the decimal logarithm of the property of interest and the measured value of its property (here the viscosity in $\mathrm{mPa} . \mathrm{s}$ ). Other data present in the file are ignored.

The following monitoring messages are displayed during this phase (suffix demol used):

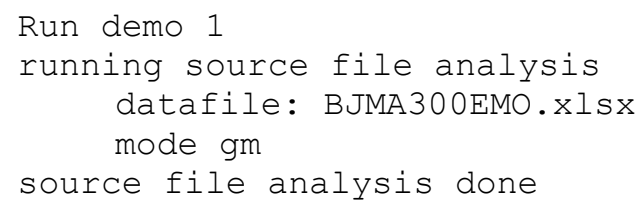

The selected mode, gm for graph machine and nn for MLP, is indicated on the fifth line. The required columns of the datafile will be used in the next step to build the appropriate models.

\section{Generation and training of the graph machines}

For each molecule, a graph machine model is automatically generated from the SMILES code. The structure of this model is derived from the 2D-structure of the molecule, and, after training, its output estimates the logarithm of the value of the selected property. All models are merged into a module that is trained with the desired property values.

The training of graph machine models, just as the training of neural networks, involves the minimization of a non-convex cost function with respect to the model parameters. Therefore, a large number of models are generated with different parameter initial values, in order to capitalize on model diversity. The chosen default number of parameter initializations, hence of generated models, is 250 . It is possible to change it, up to 1000,2000 or more, depending on the speed of the machine on which the code is executed.

After training, two important quantities are computed from each model: its root mean squared error (RMSE) and its virtual leave-one-out score (VLOO score). RMSE is the value of the cost function at the end of training; it indicates the accuracy of the estimation of the property of interest for the molecules present in the training set. The VLOO score is an estimator of the ability of the model to make accurate predictions on fresh data (not present in the training set). The training outcomes are ranked in order of increasing "VLOO score" values, since the lower that score, the better the corresponding model for prediction.

The following monitoring messages are displayed during this phase:

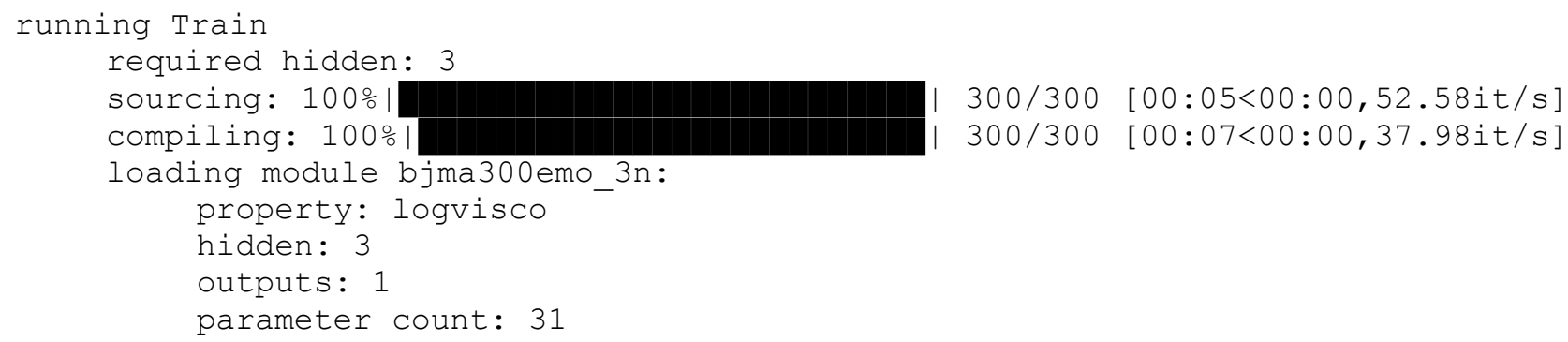


25 first training results ordered by increasing VLOO score:

ID RMSE VLOO score

$\begin{array}{lll}179 & 0.114 & 0.131\end{array}$

$\begin{array}{lll}186 & 0.107 & 0.132\end{array}$

$\begin{array}{lll}74 & 0.107 & 0.132\end{array}$

$\begin{array}{lll}98 & 0.107 & 0.133\end{array}$

$\begin{array}{lll}161 & 0.108 & 0.133\end{array}$

$\begin{array}{lll}226 & 0.114 & 0.134\end{array}$

$\begin{array}{lll}6 & 0.108 & 0.134\end{array}$

$\begin{array}{lll}113 & 0.112 & 0.135\end{array}$

$224 \quad 0.118 \quad 0.136$

$\begin{array}{lll}187 & 0.118 & 0.136\end{array}$

$\begin{array}{lll}24 & 0.101 & 0.137\end{array}$

$\begin{array}{lll}127 & 0.118 & 0.137\end{array}$

$\begin{array}{lll}149 & 0.118 & 0.137\end{array}$

$\begin{array}{lll}112 & 0.118 & 0.137\end{array}$

$\begin{array}{lll}198 & 0.115 & 0.138\end{array}$

$\begin{array}{lll}157 & 0.115 & 0.138\end{array}$

$\begin{array}{lll}172 & 0.117 & 0.138\end{array}$

$\begin{array}{lll}183 & 0.112 & 0.138\end{array}$

$\begin{array}{lll}1 & 0.115 & 0.138\end{array}$

$\begin{array}{lll}212 & 0.117 & 0.138\end{array}$

$\begin{array}{lll}145 & 0.112 & 0.139\end{array}$

$\begin{array}{lll}72 & 0.117 & 0.139\end{array}$

$\begin{array}{lll}173 & 0.117 & 0.139\end{array}$

$\begin{array}{lll}12 & 0.110 & 0.139\end{array}$

$\begin{array}{lll}123 & 0.110 & 0.139\end{array}$

Writing model file:

/Path_to_homefolder/docker/result/GM_BJMA300EMO_250T_0I_250E_25S_SEED1908839970_ $3 \mathrm{~N} \cdot \mathrm{nn} \overline{\mathrm{x}}$

Train done

Note: RMSE and VLOO score are dimensionless since the logarithm values of the viscosity are used for training; ID represents the training index considered. A file with a nnx extension is created at the end of the training process. It will be used for the test estimation of the viscosity (as a logarithm) in the next phase. If the source module used for training has already been compiled, it is loaded from a folder called workdir (located in the home folder) and the training step starts immediately.

At the beginning of each training, the model parameters are initialized with a pseudorandom number generator. The corresponding initialization data can then be recalled with the seed option. The displayed value for the seed is the seed used by random the number generator (here 1908839970).

\section{Test}

The data file used is BJMA300EMO.xlsx, and the test data are contained in the TEST cell range. These are the 22 molecules of the cosmetic oil set.

The following monitoring messages are displayed during this phase:

Running Test

test: $100 \%$

22/22 [00:01<00:00,13.59it/s ]

Test done

\section{LOO cross-validation}

300 models are trained during this last part with a number of parameter initializations equal to 25 for each model. All the generated models are kept for the estimation of the viscosity of the withdrawn molecule, The messages displayed during this phase are the following: 


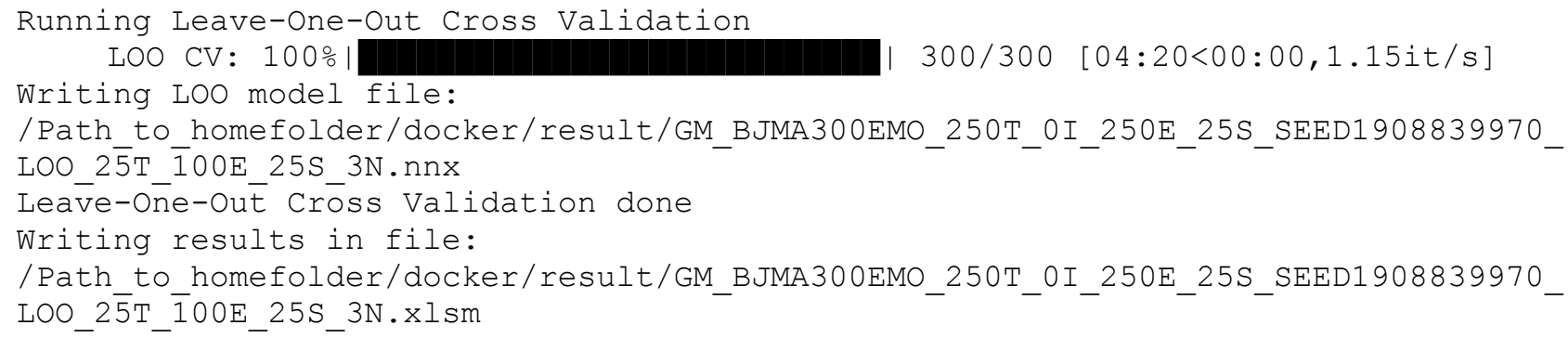

A file with an xlsm extension is created when the leave-one-out cross-validation (LOO CV) is done. It is located in a folder called result (located also in the home folder), and it contains the results in several sheets as described in next section. Some decorators are used to name this file, and their meaning is explained in the 'Advanced Options' section.

\section{Output File}

The output file has an xlsm (not xlsx) extension because it contains a macro used to close the file without getting the excel popup warning. As a result, any modification made to this file should be saved before closing. It can be read with Excel 2010-2019 (or more recent) or with LibreOffice 4.3.4 (or more recent).

The file contains eight sheets:

- Training summary;

- Training results;

- Test results;

- LOO CV results;

- Training \#xxx chart;

- VLOO \#xxx chart;

- Test result chart(yy);

- $\quad$ LOO CV chart(zz);

where $\mathrm{xxx}$ is the ID of the model that has the smallest VLOO score, yy is the number of models selected for the computation of the mean for the examples of the test (penultimate sheet) and zz is the number of models selected for the computation of the mean LOO estimations (last sheet).

\section{Training summary}

This sheet displays the results obtained with the twenty-five models having the smallest VLOO scores. The training results are ranked in order of increasing value of the VLOO score.

For each trained model, the following quantities are displayed:

- the identification number;

- the root mean square error (RMSE);

- the Virtual Leave-One-Out score (VLOO), which is an estimator of the root mean square estimation error that would be obtained by the model on molecules that are not present in the training set.

- $\quad$ the average (Avg) VLOO is, for row number $i$, the mean of the VLOO scores from row 2 to row $i$.

- the StdDev VLOO is the standard deviation of the VLOO scores from row 2 to row $i$.

\section{Training results}

This sheet displays the results for the top twenty-five trainings for each molecule (selected criterion by default: the twenty-five smallest VLOO scores).

For each molecule and each training, the following quantities are displayed:

- the value of the decimal logarithm of the measured viscosity;

- the estimated value of the logarithm of the viscosity;

- the estimation error (difference between the measured value and the estimated of the viscosity logarithm);

- the leverage; 
- the virtual leave-one-out estimation of the logarithm of the viscosity of the molecule, i.e. the estimation of the value of the logarithm of the viscosity that the model would have made if the molecule had not been present in the training set.

Below the results, the values of the following variables are displayed:

- line SUM: the sum of the leverages computed after training, equal to the number of parameters of the model;

- line VLOO score: the Virtual Leave-One-Out score computed after training;

- line RMSE: the training Root Mean Squared Error;

- line Pearson: the Pearson correlation coefficient R between the logarithms of the measured values and the estimated values;

- line $\mathrm{R}^{2}$ : the coefficient of determination, square of the Pearson coefficient $\mathrm{R}$;

\section{LOO CV results}

This sheet displays the results for all the trainings (twenty-five by default) for each molecule. For each molecule and each training, the following quantities are displayed:

- the value of the decimal logarithm of the measured viscosity;

- the mean estimated value of the decimal logarithm of the viscosity;

- the estimation error.

Below the results, the displayed value for the LOO score is the Leave-One-Out score computed after training.

\section{Test results}

The estimations made on the test set are displayed in that sheet. These values are obtained by averaging the estimations produced by the twenty-five models that have the smallest VLOO training scores.

For each molecule, the following quantities are displayed:

- the logarithm of the measured value of the viscosity;

- the mean estimated value of the logarithm of the viscosity;

- the estimation error.

Below the results, the line "Selected models" recalls the number of selected trainings used for the mean estimation.

The RMSE, Pearson coefficient, and $\mathrm{R}^{2}$, are defined as explained in the Training results subsection.

\section{Training \#xxx chart}

Scatter plot (estimated values of the logarithm of the viscosity versus logarithm of the measured values of the viscosity) of the 300 molecules of the training set for the model with ID xxx that has the smallest VLOO training score. The straight line is the bisector; this is also the case in the VLOO and test result charts described below. This is an interactive plot: when clicking on a data point a window is popping up with the following information:

- Molecule name;

- Logarithm of the measured viscosity;

- Estimated value of the viscosity;

- A question asking whether the corresponding data sheet should be opened.

If a positive answer is returned to this question, the 'Training results' sheet is opened, positioned on the line corresponding to the data point selected.

The same interactivity has been implemented for the two following charts. With the 'Test result chart', the 'Test results' sheet is opened instead of the 'Training results' sheet.

\section{VLOO \#xxx chart}

Scatter plot (VLOO-estimated values of the logarithm of the viscosity vs. logarithm of the measured values of the viscosity) of the 300 molecules of the training set for the model with ID xxx that has the smallest VLOO training score. 


\section{Test result chart(yy)}

Scatter plot (estimated values of the logarithm of the viscosity vs. logarithm of the measured values of the viscosity) of the 22 molecules of the test set. The estimated values are averaged over the number of models (yy) that have the smallest VLOO training score.

\section{LOO CV $\operatorname{chart}(\mathrm{zz})$}

Scatter plot (estimated values of the logarithm of the viscosity vs. logarithm of the measured values of the viscosity) of the 300 molecules of the complete set. The estimated values are averaged over the number of models used in the cross-validation sequence (twenty-five by default).

\section{Advanced Options}

The execution of the graph machine demonstration can be customized from the command line. The proposed default command line is (gm mode is invoked if demo1 is omitted):

docker run -it --rm -v /docker:/host espcigm/viscosity:demo

It contains the following terms:

- "docker": calls the Docker daemon of the host machine;

- "run": launches a Docker container from the Docker image;

- "-it": opens and launches the interactive mode;

- "--rm": destroys the container at the end of the session;

- "-v $\sim$ /docker:/host": creates a volume in the container, and shares it with the $\sim /$ docker tree on the host machine;

- "espcigm/viscosity:demo": name of the Docker image launched with the run command.

Parameters can be passed to the graph machine demonstrator through options that are added after the docker name and which are detailed below. To input a value for an option, the following syntax is used:

$-\mathrm{x}<$ value $>$

Where $\mathrm{x}$ denotes the code of the option command to which the value is assigned. The order of the option assignment positions in the line is meaningless.

\section{Available options}

Options available are listed below (case sensitive):

- $\mathrm{s}$ : assigns a seed provided by the pseudorandom generator during the model parameter initialization. By default, the seed is equal to 1518881864 for graph machine mode and to 155912792 for neural network mode. To have an autogenerated seed use the value $<-1>$;

- I: assigns a number of parameter initializations hence a number of trainings of the model; the default is 250 ;

- e: assigns a number of epochs for each training, the default is 250 ;

- H: assigns a number of hidden neurons; the default is 7 (GM) or 13 (MLP). This parameter may be a list in one of the formats $\mathrm{x}, \mathrm{y}, \mathrm{z}$ or $<\min >:<\max >[:<$ step $>$; default step is 1 ; in such a case, the full computation will be performed for each value of the number of hidden neurons, and a separate result file will be written;

- M: assigns a number of selected trainings (that have the smallest VLOO scores) for display and computation of the estimated values for the molecules of the test set; the default is 25 ; this parameter may be a list in one of the formats $\mathrm{x}, \mathrm{y}, \mathrm{z}$ or $\langle\min \rangle:\langle\max >:[\langle$ step $>$; default step is 1 ; in such a case, the computation will be performed for each value of the number of selected trainings, and the results will be written in the same result file;

- a: action to be performed by the run command, entered as a list of text strings separated by comma; values can be train, test, loo; default is 'train,test';

- d: a comma-separated list of decorators that are appended to the result file name; the resulting decoration strings are separated by underscores;

Decorator code

$\checkmark$ t: number of training initializations

$\checkmark$ e: number of epochs

$\checkmark$ n: number of hidden neurons
Suffix appended

" $<$ value $>$ T"

" $<$ value $>$ E"

"<value $>\mathrm{N}$ " 
$\checkmark$ s: number of selected models (smallest VLOOs)

$\checkmark$ se: value of the seed used for pseudorandom generator i: number of input descriptors
" $<$ value $>$ S"

"SEED $<$ value $>$ "

" $<$ value $>$ I"

Example of result file name: NN_BJMA300EMO_250T_5I_250E_25S_SEED155912792_13N

By default, graph machine provides a full list of decorators.

Some special options give only information, and are typed at the end of the docker command line:

- help: the help file is displayed;

- version: the software version numbers are displayed;

- license: the license text is displayed.

\section{Some examples of command line}

Display the help file:

docker run -it --rm $-\mathrm{v} \sim /$ docker:/host espcigm/viscosity:demo help

Use an auto-generated seed and 3 hidden neurons:

docker run -it --rm $-\mathrm{v} \sim /$ docker:/host espcigm/viscosity:demo demol $-\mathrm{s}-1-\mathrm{l} \quad 3$

Use 1947 as random seed and 50 initializations:

docker run -it --rm -v /docker:/host espcigm/viscosity:demo demol -s 1947 -I 50

Run a graph machine loo sequence in automated mode with the value 224059737 as the random seed, with 7 hidden neurons, 25 models (i.e. 25 different parameter initializations), each training running 100 epochs:

docker run -it --rm -v /docker:/host espcigm/viscosity:demo demo1 -a $100-\mathrm{H} 7$ s 224059737 -I 25 -e 100

Run a graph machine train+test sequence in automated mode with the value 1518881864 as the random seed, and 2 to 10 hidden neurons, the nine training modules created being accessible to the host machine to be reused in future sessions:

docker run -it --rm -v /docker:/host espcigm/viscosity:demo demo1 -H 2:10 -s 1518881864

After completion of the computations, nine files with the names 'BJMA300EMO_250T_0I 250E_ 25S_SEED1518881864_xN', where $\mathrm{x}$ varies from 2 to 10 are written in a directory created in the $\sim /$ docker/result directory. A supplementary file with the name 'GM_BJMA300EMO_RECAP_250T_0I_250E_25S SEED1518881864_2--10N' is also written. It contains a line chart displaying the variation of the average VLOO score versus the number of hidden neurons. 


\section{GRAPH MACHINE RESULTS WITH DOCKER}

All computations were made on a $3 \mathrm{GHz} 8$-Core Intel Xeon E5 Mac Pro with $32 \mathrm{~GB}$ of RAM running macOs High Sierra 10.13.6 (Docker configuration: 16 CPUs, 28 GB allocated RAM). All graph machine tasks were launched with the maximum number of available CPUs minus 2, i.e. 14 CPUs on the Mac Pro.

The training data used for all computations, contained in the DATA cell range, are the names, the SMILES codes, the five $\sigma$-moments of the Klamt set, and the logarithm of the measured viscosity values for the 300 molecules of the training set. The test data contained in the TEST cell range are those of the 22 molecules of the cosmetic oil test set.

\section{Selection of the graph machine complexity with the training set of $\mathbf{3 0 0}$ molecules.}

\section{LOO score selection}

Five different initializations were randomly launched for all complexities in the case of LOO. To shorten the training time, the number of epochs was taken equal to 100 and the number of initializations to 25 . The results are given in the following table; for each complexity the LOO scores (LOOs) are displayed for the five initializations launched.

\begin{tabular}{ccccccccccc}
\hline Complexity & Seed & LOOs & Seed & LOOs & Seed & LOOs & Seed & LOOs & Seed & LOOs \\
\hline $2 \mathrm{n}$ & 1598080059 & 0.252 & 400885234 & 0.254 & 1998823220 & 0.254 & 2502839077 & 0.258 & 929741247 & 0.259 \\
\hline $3 \mathrm{n}$ & 2415294752 & 0.156 & 2241153473 & 0.158 & 2416826852 & 0.150 & 4048619328 & 0.152 & 301885405 & 0.156 \\
\hline $4 \mathrm{n}$ & 1871671919 & 0.128 & 1278337646 & 0.128 & 258455810 & 0.135 & 2244962976 & 0.132 & 1460691685 & 0.133 \\
\hline $5 \mathrm{n}$ & 2304338237 & 0.120 & 4115772207 & 0.122 & 2596565213 & 0.119 & 768281810 & 0.122 & 908609374 & 0.121 \\
\hline $6 \mathrm{n}$ & 2685244193 & 0.118 & 414539629 & 0.119 & 1052302713 & 0.116 & 3017552361 & 0.121 & 4274744077 & 0.110 \\
\hline $7 \mathrm{n}$ & 224059737 & 0.113 & 771320915 & 0.113 & 4269743458 & 0.114 & 2761458253 & 0.114 & 3868870568 & 0.113 \\
\hline $8 \mathrm{n}$ & 1908839970 & 0.110 & 3809503575 & 0.115 & 1236143821 & 0.108 & 1867498199 & 0.108 & 337016226 & 0.107 \\
\hline $9 \mathrm{n}$ & 1684822067 & 0.106 & 1897267007 & 0.112 & 318343767 & 0.107 & 1199291260 & 0.112 & 2323709732 & 0.111 \\
\hline $10 \mathrm{n}$ & 1561791813 & 0.111 & 1588805695 & 0.110 & 1477466720 & 0.112 & 342959128 & 0.111 & 1462341980 & 0.107 \\
\hline
\end{tabular}

The computations can be easily repeated, thanks to the seed values. For example, in the case of the eight hidden neuron complexity, the command line to get the LOO score value highlighted in green is the following:

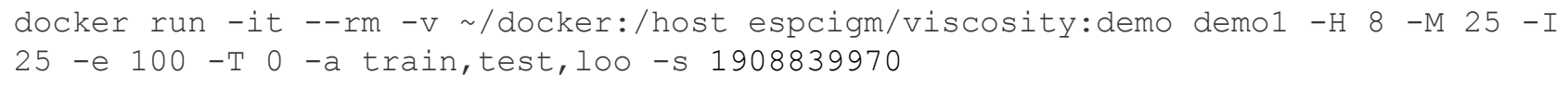

This computation might take some time; on the Mac Pro used, it was completed within 1 hour and 31 minutes. When the mean of the five LOO scores given in the previous table is plotted versus the number of hidden neurons, the following plot is obtained:

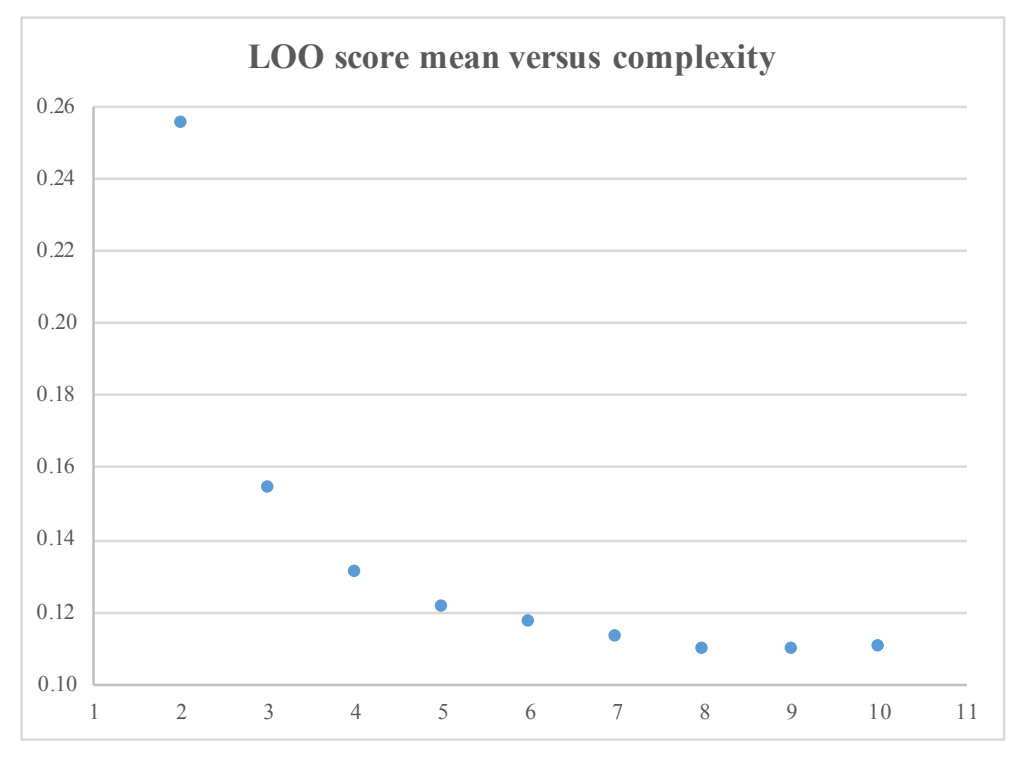


Since no decrease of the LOO score mean is observed above eight hidden neurons, the eight hidden neurons model is kept for estimations of the viscosity of new molecules.

\section{VLOO score selection}

To select the appropriate complexity for the graph machines, the means of the VLOO scores of the 25 models (out of 250) that have the smallest VLOO scores are compared when the number of hidden neurons increases. The Docker run command is the following, the number of hidden neurons being varied from 2 to 10 , and the seed value being equal to 2678535059 :

docker run -it --rm -v /docker:/host espcigm/viscosity:demo demo1 -H 2:10 -s 2678535059

The main results are the following (nine files are written in the $\sim /$ docker/result directory):

\begin{tabular}{cccc}
\hline $\begin{array}{c}\text { Number of hidden } \\
\text { neurons }\end{array}$ & $\begin{array}{c}\text { Best VLOO score } \\
\text { mean (25 models) }\end{array}$ & $\begin{array}{c}\text { Smallest } \\
\text { RMSTE }\end{array}$ & $\begin{array}{c}\text { Smallest } \\
\text { VLOO score }\end{array}$ \\
\hline 2 & 0.157 & 0.140 & 0.154 \\
3 & 0.135 & 0.101 & 0.131 \\
4 & 0.124 & 0.082 & 0.114 \\
5 & 0.112 & 0.066 & 0.093 \\
6 & 0.105 & 0.051 & 0.084 \\
7 & 0.100 & 0.045 & 0.084 \\
8 & 0.096 & 0.035 & 0.081 \\
9 & 0.101 & 0.025 & 0.071 \\
10 & 0.123 & 0.019 & 0.070 \\
\hline
\end{tabular}

The corresponding chart given in the RECAP file is the following (displayed for VLOO scores only):

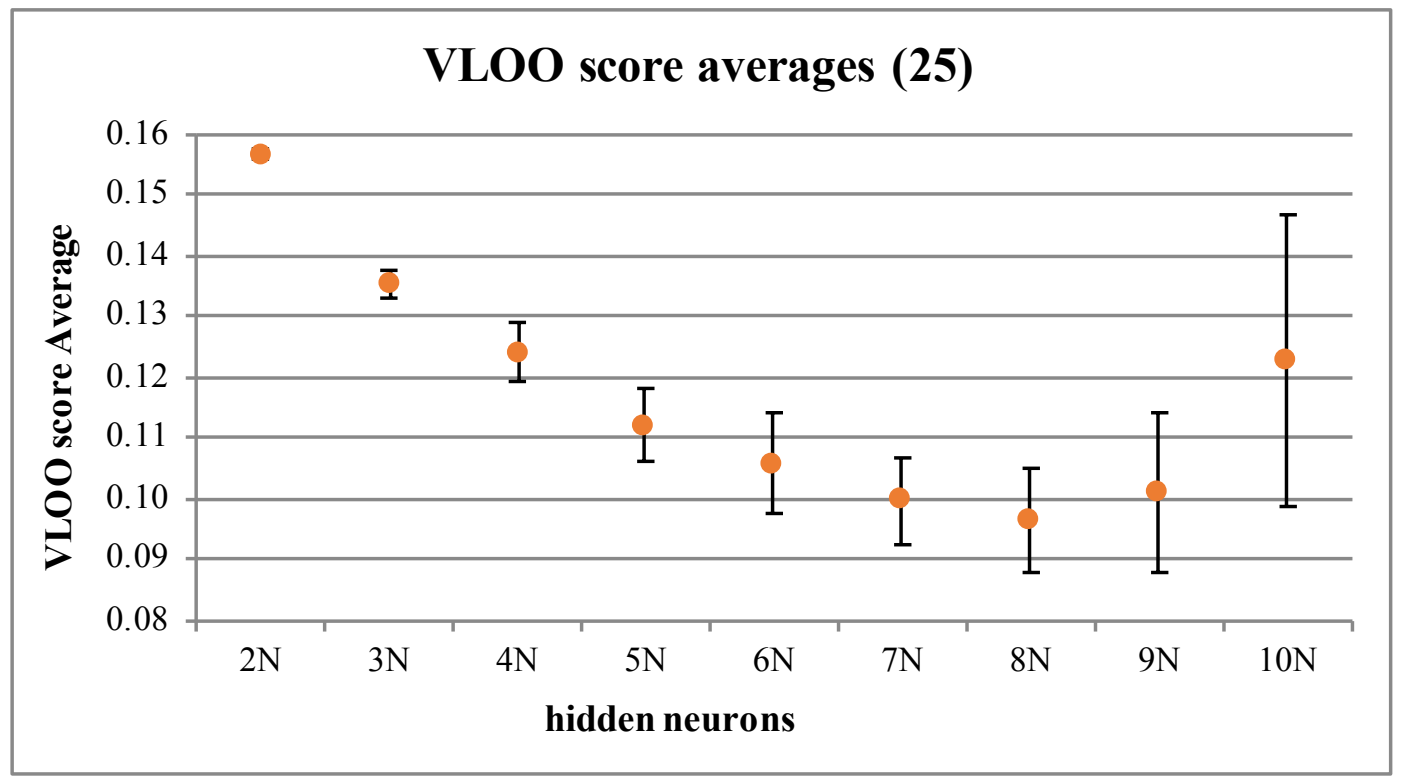

Thus, given the available data, eight hidden neurons is the complexity that is expected to provide the models that generalize best.

\section{Selection of the MLP complexity with the training set of 300 molecules}

\section{VLOO score selection}

To select the appropriate complexity for the MLP approach, the mean of the VLOO scores of the 25 models (out of 250) that have the smallest VLOO scores are compared when the number of hidden neurons increases. The Docker run command is the following, the number of hidden neurons being varied in this case from 2 to 20 :

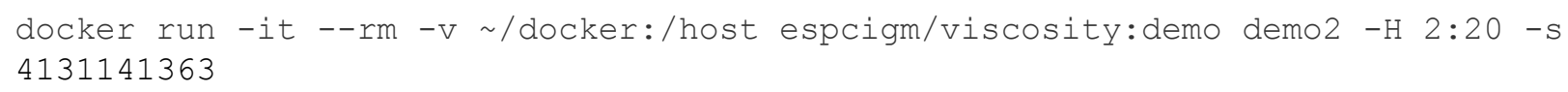

The main results are the following (nineteen files are written in the directory with the name BJMA300EMO- 
SEED4131141363-2--20N created in the $\sim /$ docker/result directory):

\begin{tabular}{cccc}
\hline $\begin{array}{c}\text { Number of hidden } \\
\text { neurons }\end{array}$ & $\begin{array}{c}\text { Best VLOO score } \\
\text { mean (25 models) }\end{array}$ & Smallest RMSTE & $\begin{array}{c}\text { Smallest } \\
\text { VLOO score }\end{array}$ \\
\hline 2 & 0.209 & 0.194 & 0.209 \\
3 & 0.192 & 0.160 & 0.190 \\
4 & 0.171 & 0.142 & 0.166 \\
5 & 0.162 & 0.127 & 0.153 \\
6 & 0.153 & 0.109 & 0.143 \\
7 & 0.148 & 0.101 & 0.140 \\
8 & 0.138 & 0.096 & 0.122 \\
9 & 0.138 & 0.089 & 0.126 \\
10 & 0.131 & 0.082 & 0.116 \\
11 & 0.133 & 0.076 & 0.120 \\
12 & 0.131 & 0.074 & 0.108 \\
13 & 0.126 & 0.069 & 0.114 \\
14 & 0.126 & 0.064 & 0.105 \\
15 & 0.129 & 0.060 & 0.119 \\
16 & 0.126 & 0.054 & 0.112 \\
17 & 0.127 & 0.054 & 0.101 \\
18 & 0.127 & 0.050 & 0.113 \\
19 & 0.129 & 0.048 & 0.100 \\
20 & 0.130 & 0.047 & 0.111 \\
\hline
\end{tabular}

The corresponding chart given in the RECAP file is the following (displayed for VLOO scores only):

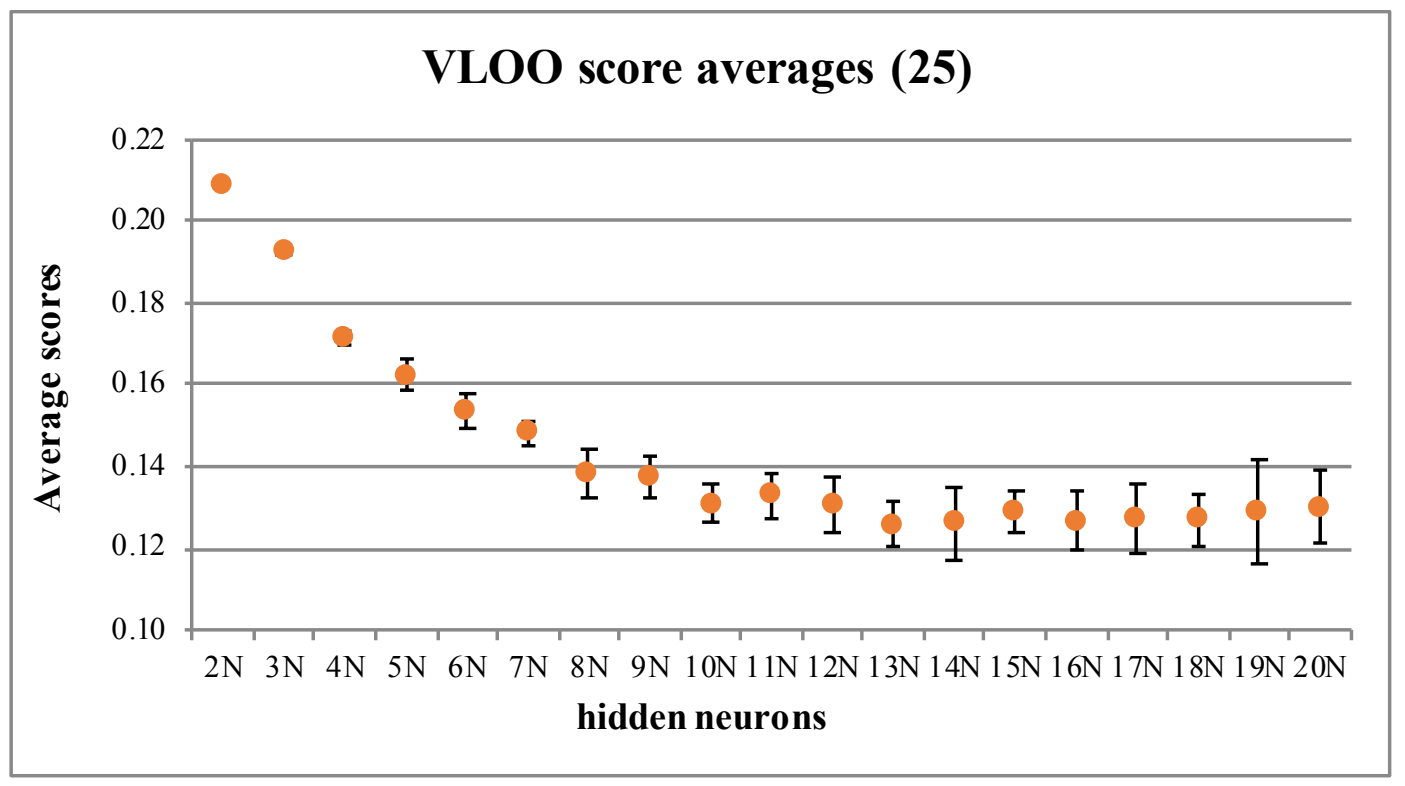

The thirteen hidden neurons model is then the best model for generalization.

\section{Estimations of the viscosity values for the 22 cosmetic oil set}

For the selected GM and MLP models, ten successive computations were launched with random seeds, respectively with the following commands:

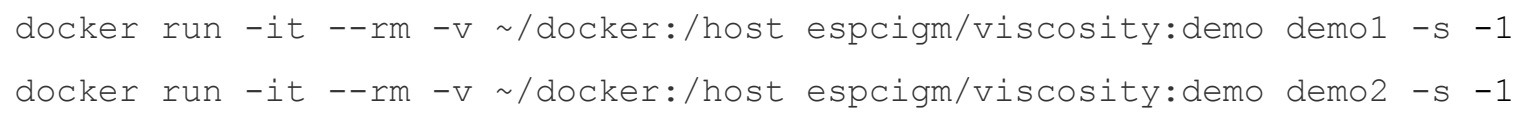

The VLOO score means on the 25 models (out of 250) that have the smallest VLOO scores were then compared for the 10 computations, and the computation that has the smaller VLOO score average was chosen for estimating the viscosity values of molecules of the test set. The results are gathered in the following table for both models. All the displayed VLOO score mean values can be re-computed by replacing, in the previous command lines, the value -1 by the appropriate seed value. 


\begin{tabular}{cc|cc}
\hline \multicolumn{2}{c|}{ GM 8n (demo1) } & \multicolumn{2}{c}{ MLP 13n (demo2) } \\
\hline Seed & VLOO score mean & Seed & VLOO score mean \\
\hline 589218094 & 0.097 & 1497695456 & 0.126 \\
1518881864 & 0.089 & 3677396744 & 0.128 \\
2030866222 & 0.099 & 2226710055 & 0.129 \\
2123925473 & 0.095 & 1157351219 & 0.131 \\
2295858324 & 0.099 & 1794690070 & 0.131 \\
2701033301 & 0.097 & 1011677354 & 0.129 \\
3216083470 & 0.094 & 1848536120 & 0.131 \\
3486587315 & 0.096 & 155912792 & 0.125 \\
3980242547 & 0.097 & 3913564510 & 0.127 \\
4065260821 & 0.102 & 2537504129 & 0.126 \\
\hline
\end{tabular}

The models that are kept are those used in the demo image, i.e. the graph machine model with eight hidden neurons seeded with the number 1518881864, and the multilayer Perceptron with thirteen hidden neurons seeded with the number 155912792. The estimated viscosity values computed with these two models for the molecules of the cosmetic oil set are those of the columns 5 and 6 of table S6. The estimated viscosity values obtained with these models for the 300 molecules of the training set are displayed as their logarithm in the columns 5 and 6 of table S5.

\section{Estimations of the viscosity for a single molecule}

Estimation of the viscosity of the molecules of the complete set or of the test set can be replicated for the default graph-machine-based model and for the default MLP based models. For example, with isopropyl isostearate (Table 3 of the paper, entry 19), whose SMILES code and sigma-moments are from Table 2 (entry 21) and Table 4 respectively, the commands are the following:

$$
\begin{aligned}
& \text { docker run -it --rm espcigm/viscosity:demo get ' } \mathrm{CC}(\mathrm{C}) \mathrm{CCCCCCCCCCCCCCC}(\mathrm{OC}(\mathrm{C}) \mathrm{C})=\mathrm{O}^{\prime} \\
& \text { docker run -it --rm espcigm/viscosity:demo get } 466.5658 .5931 .513 .010 .00
\end{aligned}
$$

The messages returned for both commands are the following:

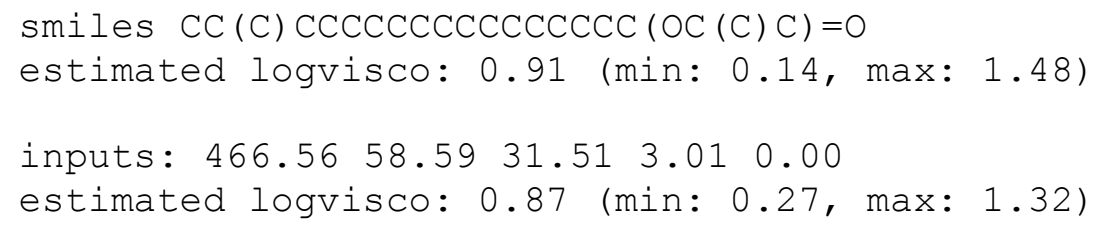

The decimal logarithm of the values of the estimated viscosity for isopropyl isostearate are equal to 0.91 (8.1 mPa.s) for the GM method, and 0.87 (7.4 mPa.s) for the MLP method. Between parentheses are given the smallest and the largest estimated values computed by the 25 models (out of 250) that have the smallest VLOO scores.

Estimation of the viscosity of any liquid of molecule containing carbon, hydrogen, oxygen and silicon atoms can also be computed starting from its SMILES code or its COSMO-RS $\sigma$-moments with similar command lines. 